\title{
Equation of state in $2+1$ flavor QCD at high temperatures
}

\author{
A. Bazavov, ${ }^{1}$ P. Petreczky, ${ }^{2}$ and J. H. Weber ${ }^{3,4}$ \\ ${ }^{1}$ Department of Computational Mathematics, Science and Engineering \\ and Department of Physics and Astronomy, Michigan State University, East Lansing, Michigan 48824, USA \\ ${ }^{2}$ Physics Department, Brookhaven National Laboratory, Upton, New York 11973, USA \\ ${ }^{3}$ Physik Department, Technische Universität München, D-85748 Garching, Germany \\ ${ }^{4}$ Exzellenzcluster Universe, Technische Universität München, D-85748 Garching, Germany
}

(Received 20 October 2017; published 31 January 2018)

\begin{abstract}
We calculate the equation of state at high temperatures in $2+1$ flavor QCD using the highly improved staggered quark (HISQ) action. We study the lattice spacing dependence of the pressure at high temperatures using lattices with temporal extent $N_{\tau}=6,8,10$ and 12 and perform continuum extrapolations. We also give a continuum estimate for the equation of state up to temperatures $T=2 \mathrm{GeV}$, which are then compared with results of the weak-coupling calculations. We find a reasonably good agreement with the weak-coupling calculations at the highest temperatures.
\end{abstract}

DOI: 10.1103/PhysRevD.97.014510

\section{INTRODUCTION}

Over the last several years there was a focused effort to calculate the equation of state of strongly interacting matter at net zero baryon density in lattice QCD using physical or nearly physical quark masses and improved staggered action [1-8]. As the result the continuum extrapolated equation of state (EoS) has been obtained in $2+1$ flavor QCD for physical light and strange quark masses $[7,8]$. The calculations have been performed using two different improved staggered discretization schemes, the so-called stout action and the highly improved staggered quark (HISQ) action. These calculations cover a temperature range up to $T=400-500 \mathrm{MeV}$. Overall the results of these calculations agree well, except for the highest temperatures, where tension between the two results can be seen [8]. It is important to clarify if this tension is just due to some statistical fluctuations or part of a systematic trend. Furthermore, for the comparison with the weak-coupling results it is highly desirable to extend the EoS calculations to higher temperatures. At temperatures $T>400 \mathrm{MeV}$ the charm quark contributes significantly to thermodynamic quantities and has to be included in the calculations [9]. Thus, one has to perform the calculations of the EoS in $2+1+1$ flavor QCD. However, in the weak-coupling calculations the inclusion of the charm quark complicates the analysis, and the effects of the charm quark on the

Published by the American Physical Society under the terms of the Creative Commons Attribution 4.0 International license. Further distribution of this work must maintain attribution to the author(s) and the published article's title, journal citation, and DOI. Funded by SCOAP ${ }^{3}$. thermodynamic quantities are only known up to next-toleading order (NLO) [10]. Also it is more difficult to control the discretization effects in the presence of the charm quark due to its large mass. Therefore, for comparison of the lattice QCD results and the weak-coupling results it is advantageous to consider thermodynamic quantities in $2+1$ flavor QCD at higher temperatures. Such calculations also provide a solid reference point for estimating the charm quark contribution to QCD thermodynamics.

The purpose of this work is to extend the calculations presented in Ref. [8] to higher temperatures. As in Ref. [8] the HISQ action will be used together with the physical value of the strange quark mass, $m_{s}$. The lattice spacing (cutoff) dependence of the pressure will be studied in detail. In the previous studies the continuum extrapolations have been performed for the trace anomaly; the pressure and other thermodynamic properties have been obtained from the trace anomaly using the integral method [11]. The cutoff dependence of the trace anomaly, however, is expected to be more complicated than the cutoff dependence of the pressure. The reason for this is the following. In the weak-coupling picture the trace anomaly receives contributions starting at three loop, i.e. at order $\alpha_{s}^{2}$. Therefore, the understanding of the cutoff dependence of the trace anomaly at high temperature would in principle require a three-loop calculation in lattice perturbation theory. This is clearly formidable task. On the other hand the pressure at high temperature receives the leading contribution at one loop $\left(\mathcal{O}\left(\alpha_{s}^{0}\right)\right)$ corresponding to the ideal gas limit. Therefore, the cutoff dependence of the pressure at high temperature is known and to fairly good approximation is described by the free gas $[12,13]$. 
For better understanding of the cutoff dependence of the EoS at high temperatures and a better control of the continuum extrapolation it is desirable to study the cutoff dependence of the pressure directly. This may also help to understand the difference between the continuumextrapolated results and the results obtained with $\mathrm{p} 4$ or asqtad-improved staggered actions and $N_{\tau}=6$ and 8 at high temperatures $[3,4]$ since cutoff effects here should be small.

It is expected that thermodynamic properties are not sensitive to the value of the light quark masses at high temperatures. The quark mass dependence of the EoS was studied in Ref. [6] and it was found that for light quark masses smaller than $0.4 m_{s}$ the quark mass dependence is very small for $T>450 \mathrm{MeV}$. Therefore, we consider light quark masses which are five times smaller than the strange quark mass, $m_{l}=m_{s} / 5$, instead of the physical value. This choice of the light quark mass corresponds to a pion mass of about $320 \mathrm{MeV}$ in the continuum limit.

The rest of the paper is organized as follows. In Sec. II we discuss details of the lattice calculations. In Sec. III we show our results for the trace anomaly. In Sec. IV we present the calculation of the pressure and its cutoff dependence. Comparison of the lattice calculations to the weak-coupling results is discussed in Sec. V. Finally Sec. VI contains our conclusions. Some technical aspects of the calculations are presented in the Appendices.

\section{LATTICE CALCULATIONS AT ZERO TEMPERATURE}

The goal of this paper is to extend the calculations of the QCD equation of state in Ref. [8] to higher temperatures. Therefore, as in Ref. [8] we use tree-level improved gauge action and HISQ action for quarks. To calculate the EoS gauge configurations at zero temperature had to be generated to perform the subtraction of the UV divergences in the thermodynamic quantities as well for the determination of the lattice spacing. We generated the gauge configurations at $T=0$ using the rational hybrid Monte-Carlo (RHMC) algorithm at five values of the lattice gauge coupling $\beta=10 / g^{2}$. The parameters of the simulations are shown in Tab. I, including the lattice volume. The lowest two $\beta$ values will be used for the purpose of comparison with the previous $2+1$ flavor results at smaller light quark masses [8], enabling us to quantify the quark

TABLE I. The parameters of the $T=0$ simulations.

\begin{tabular}{lcccc}
\hline \hline$\beta$ & $m_{s}$ & vol & a [fm] & Number of traj. \\
\hline 7.030 & 0.03560 & $48^{4}$ & 0.08253 & 1890 \\
7.825 & 0.01542 & $64^{4}$ & 0.04036 & 1265 \\
8.000 & 0.01299 & $64^{4}$ & 0.03469 & 3927 \\
8.200 & 0.01071 & $64^{4}$ & 0.02924 & 3927 \\
8.400 & 0.00887 & $64^{4}$ & 0.02467 & 3927 \\
\hline \hline
\end{tabular}

mass effects in the scale setting procedure as well as in the thermodynamic quantities.

The lattice spacings corresponding to the highest three $\beta$ values in Table I are smaller than $0.035 \mathrm{fm}$. At these small lattice spacings it is expected that the Monte-Carlo (MC) evolution of the topological charge will effectively freeze. Indeed, we observe that the topological charge does not change in the MC evolution. To deal with this problem we generated MC streams corresponding to different values of topological charge, namely $Q=0,1$ and 2 . We checked whether the observables of interest are sensitive to the value of the topological charge, but we did not find any sensitivity. The dependence of different observables on the topological charge is discussed in Appendix A.

To determine the lattice spacing we calculated the static quark antiquark potential. The lattice spacing is determined through the scale parameters $r_{1}$ and $r_{2}$ defined as

$$
\left.r^{2} \frac{d V(r)}{d r}\right|_{r=r_{1}}=1,\left.\quad r^{2} \frac{d V}{d r}\right|_{r=r_{2}}=\frac{1}{2} .
$$

The parameter $r_{1}$ is widely used by the MILC and HotQCD collaborations to set the lattice spacing (see e.g. Ref. [8]). The value of this parameter is $r_{1}=0.3106 \mathrm{fm}$ [14]. Since we consider smaller lattice spacings it is useful to consider the scale parameter $r_{2}$. The calculation of the static potential and the determination of $r_{1}$ and $r_{2}$ scales is discussed in Appendix A.

For the two lower $\beta$ values in Table I we could compare the results on the static potential calculated for $m_{l}=m_{s} / 5$ with the previous calculations performed at $m_{l}=m_{s} / 20$ to study quark mass effects. We find no quark mass effects at the shortest distances. Quark mass effects increase with increasing distances but are less then $0.2 \%$ for $r<r_{1}$. At distances around $r=r_{1}$ the statistical errors in the static potential are large enough so that no quark mass effects in the derivative of the potential can be seen. Therefore, we can combine the newly determined values of $r_{1}$ with the previously published HotQCD results to obtain $r_{1} / a$ as function of $\beta$. The details of this analysis are given in Appendix A.

\section{THE QCD TRACE ANOMALY}

To extend the calculation of the equation of state of $2+1$ flavor QCD we used the integral method, which relies on the calculation of the trace of the energy momentum tensor $\Theta^{\mu \mu}=\epsilon-3 p$ or the trace anomaly for short $[3,4]$. The pressure can be calculated in terms of the trace anomaly as follows:

$$
\frac{p(T)}{T^{4}}-\frac{p\left(T_{0}\right)}{T_{0}^{4}}=\int_{T_{0}}^{T} d T^{\prime} \frac{\epsilon-3 p}{T^{\prime 5}},
$$

where $T_{0}$ is some reference temperature, which is sufficiently small, so $p\left(T_{0}\right)$ can be either set to zero or taken 

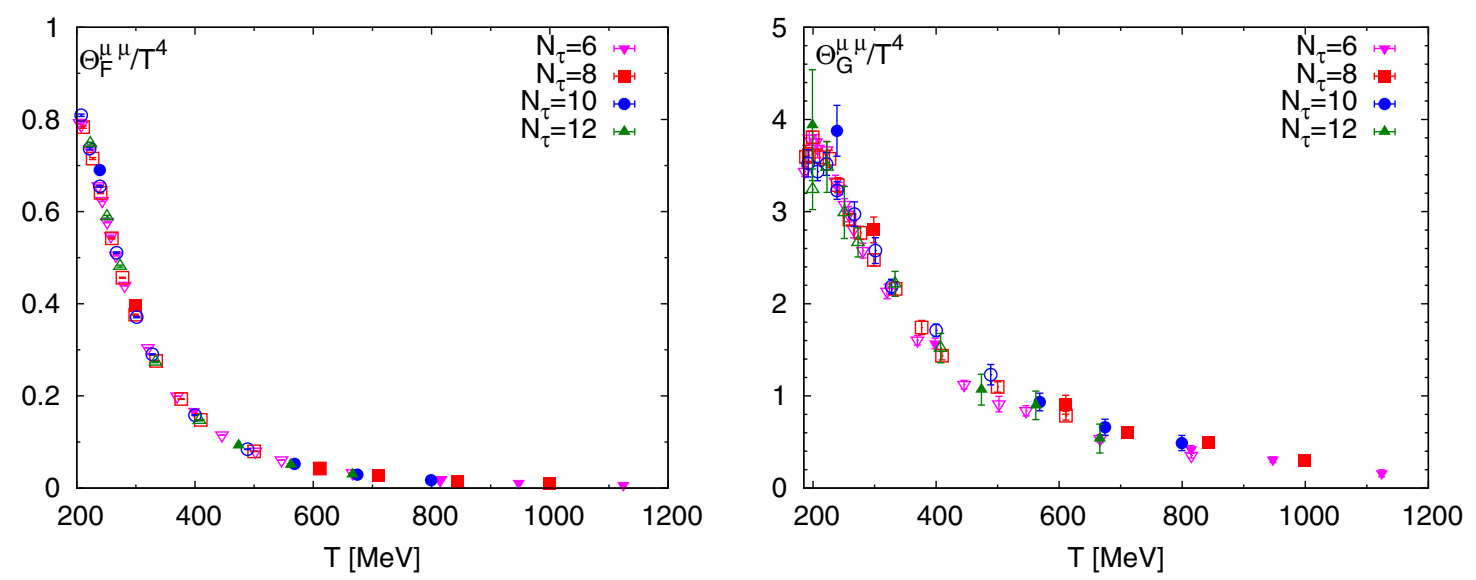

FIG. 1. The fermionic part (left) and the gauge part (right) of the trace anomaly obtained with HISQ action. The open symbols correspond to the HotQCD results for $m_{l}=m_{s} / 20$ [8].

from the hadron resonance gas calculation $[3,4,8]$. The trace anomaly can be expressed in terms of the expectation values of the gauge action, $\left\langle s_{G}\right\rangle_{\tau(0)}$, and the light, $\langle\bar{\psi} \psi\rangle_{l, \tau(0)}$, and strange, $\langle\bar{\psi} \psi\rangle_{s, \tau(0)}$, quark condensates, calculated at finite and zero temperature, respectively. For the HISQ action the corresponding formula has the form [8]:

$$
\begin{gathered}
\frac{\epsilon-3 p}{T^{4}} \equiv \frac{\Theta_{G}^{\mu \mu}(T)}{T^{4}}+\frac{\Theta_{F}^{\mu \mu}(T)}{T^{4}}, \\
\frac{\Theta_{G}^{\mu \mu}(T)}{T^{4}}=R_{\beta}\left[\left\langle s_{G}\right\rangle_{0}-\left\langle s_{G}\right\rangle_{\tau}\right] N_{\tau}^{4}, \\
\frac{\Theta_{F}^{\mu \mu}(T)}{T^{4}}=-R_{\beta} R_{m}\left[2 m_{l}\left(\langle\bar{\psi} \psi\rangle_{l, 0}-\langle\bar{\psi} \psi\rangle_{l, \tau}\right)\right. \\
\left.+m_{s}\left(\langle\bar{\psi} \psi\rangle_{s, 0}-\langle\bar{\psi} \psi\rangle_{s, \tau}\right)\right] N_{\tau}^{4} .
\end{gathered}
$$

Here we used the same notation as in Ref. [8] and we made explicit the separation of the trace anomaly into the fermionic and gluonic parts. Furthermore, we introduced the nonperturbative beta function and mass renormalization function defined as $[3,4]$

$$
\begin{gathered}
R_{\beta}(\beta)=\frac{r_{1}}{a}\left(\frac{\mathrm{d}\left(r_{1} / a\right)}{\mathrm{d} \beta}\right)^{-1}, \\
R_{m}(\beta)=\frac{1}{m_{s}(\beta)} \frac{\mathrm{d} m_{s}(\beta)}{\mathrm{d} \beta} .
\end{gathered}
$$

The calculation of the nonperturbative beta function is discussed in Appendix A. The mass renormalization function is taken from Ref. [8]. As also discussed in Appendix A, the new zero temperature calculations are consistent with this mass renormalization function.

To calculate the trace anomaly at temperatures corresponding to the values of $\beta$ given in Table I we use the finite temperature gauge configurations from the TUMQCD collaboration $[15,16]$. These gauge configurations have been generated on $N_{\sigma}^{3} \times N_{\tau}$ lattices with $N_{\tau}=4,6,8,10$ and 12 and $N_{\sigma}=4 N_{\tau}$. The maximal temperature corresponding to these lattices is about $2 \mathrm{GeV}$.

Now we will discuss our numerical results on the trace anomaly, in particular, its dependence on the light quark masses. There are two sources of quark mass dependence of the trace anomaly. First, is the dependence of the trace anomaly on the light sea quark masses. The second is the explicit dependence of the fermionic part of the trace anomaly on the light quark mass. As we will see later there are also differences in the cutoff $\left(N_{\tau}\right)$ dependence of the fermionic and gluonic parts of the trace anomaly. Therefore, in the following we will discuss the numerical results for $\Theta_{F}^{\mu \mu}$ and $\Theta_{G}^{\mu \mu}$ separately. The fermionic part of the trace anomaly, $\Theta_{F}^{\mu \mu}$ is shown in Fig. 1 (left) and compared with the published HotQCD results obtained for $m_{l}=$ $m_{s} / 20$ [8] and shown as open symbols. To take into account the explicit dependence on the light quark masses in the calculation of $\Theta_{F}^{\mu \mu}$ we used the value $m_{l}=m_{s} / 20$ instead of $m_{l}=m_{s} / 5$. We see from the figure that after adjusting the light quark mass there is no quark mass dependence in $\Theta_{F}^{\mu \mu}$ for $T>300 \mathrm{MeV}$, i.e. the quark mass dependence of $\Theta_{F}^{\mu \mu}$ due to the sea quarks is very small. From Fig. 1 (left) we also see that the cutoff effects in $\Theta_{F}^{\mu \mu}$ are very small in accordance with the previous study [8]. Finally, we note that statistical errors for $\Theta_{F}^{\mu \mu}$ are tiny. Our results for the gluonic part of the trace anomaly, $\Theta_{G}^{\mu \mu}$, are shown in Fig. 1(right). The cutoff and quark mass dependence of $\Theta_{G}^{\mu \mu}$ can be clearly seen. The quark mass dependence of $\Theta_{G}^{\mu \mu}$ is due to the sea quarks and thus cannot be corrected. It is the sole source of the quark mass dependence of the trace anomaly shown in Fig. 2. We see, however, that quark mass effects become smaller at high temperatures and statistically are not significant for $T>400 \mathrm{MeV}$. The statistical errors for $\Theta_{G}^{\mu \mu}$ are much larger than for $\Theta_{F}^{\mu \mu}$ and it is the dominant contribution to the trace anomaly. 


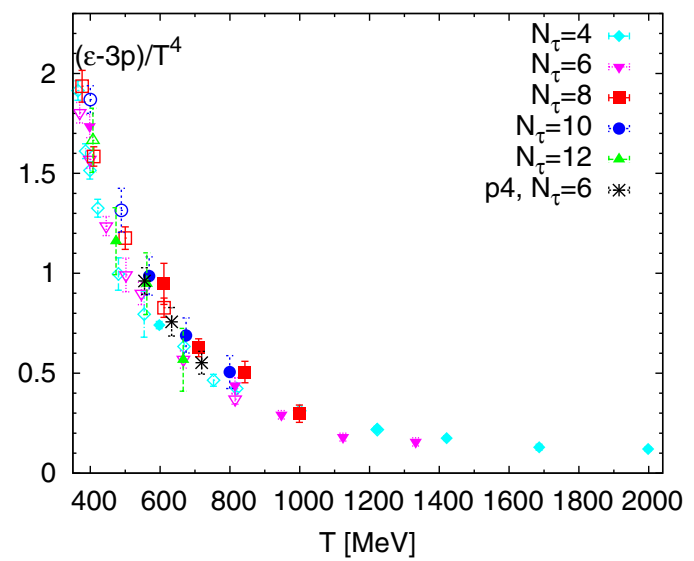

FIG. 2. The numerical results for the trace anomaly at two different quark masses (see text). The open symbols correspond to $m_{l} / m_{s}=1 / 20$, while the filled symbols correspond to $m_{l} / m_{s}=1 / 5$. The bursts correspond to calculations with $\mathrm{p} 4$ action and $m_{l}=m_{s} / 10$ [3].

For the calculation of the trace anomaly at high temperatures we also used $N_{\tau}=4$ lattices from the TUMQCD collaboration obtained with $m_{l}=m_{s} / 20$ [15] and $m_{l}=$ $m_{s} / 5$ [16]. Our results for the trace anomaly at high temperatures are summarized in Fig. 2. The open symbols in the figure refer to $m_{l} / m_{s}=1 / 20$ results, while the filled symbols refer to $m_{l} / m_{s}=1 / 5$ results. All the $m_{l}=m_{s} / 20$ results for $\epsilon-3 p$ are from Ref. [8], except the ones for $N_{\tau}=4$ and those for $N_{\tau}=6$ with $\beta=7.03$ or 7.825 . From Fig. 2 we see that $m_{s} / 20$ results smoothly match to the $m_{s} / 5$ results at high temperatures. This is expected. From the calculations of the trace anomaly performed with stout action at several quark masses we can estimate that the difference in the trace anomaly calculated for $m_{l}=m_{s} / 5$ and $m_{l}=m_{s} / 20$ is $10 \%, 4 \%, 3 \%$ and $<1 \%$ for $T=300$, 400, 500 and $600 \mathrm{MeV}$, respectively. Our calculations with $m_{l}=m_{s} / 5$ at $T \leq 400 \mathrm{MeV}$ confirm these expectations. The statistical errors shown in Fig. 2 are much larger than the above differences in the temperature range of interest, so no quark mass effects are visible given the errors. In Fig. 2 we also show the trace anomaly calculated with $\mathrm{p} 4$ action for $m_{l} / m_{s}=1 / 10$ and $N_{\tau}=6$ [3]. The corresponding results agree well with the HISQ results. Overall we see that quark mass effects are very small at high temperatures and therefore it is justified to study QCD thermodynamics with $m_{l}=m_{s} / 5$ in this region. Finally, we note that there is no visible cutoff dependence for $\epsilon-3 p$ for $N_{\tau} \geq 8$ in the high temperature region, while the $N_{\tau}=4$ and 6 data are systematically below the $N_{\tau} \geq 8$ results.

While the main purpose of this work is to extend the EoS calculations to high temperatures we also revisited the trace anomaly in the low temperature region for $N_{\tau}=10,12$ using the gauge configurations generated by the TUMQCD collaboration for the study of the Polyakov loop [15]. The reason behind this is the fact that unlike in Ref. [8] the

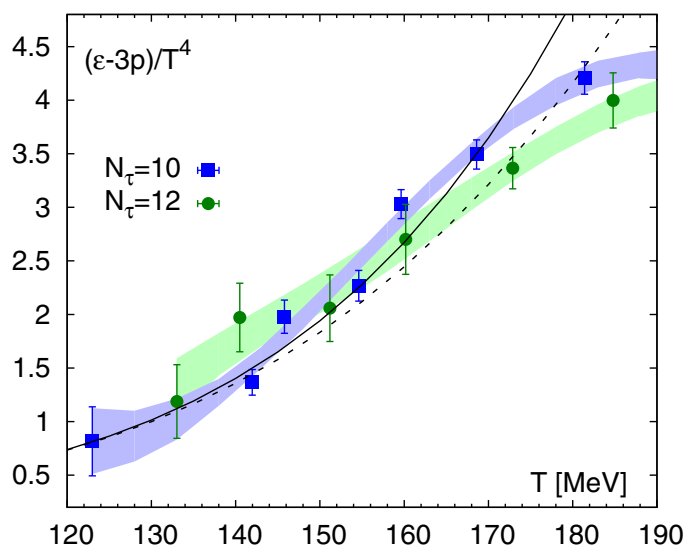

FIG. 3. The trace anomaly calculated in the low temperature region using $N_{\tau}=10$ and $N_{\tau}=12$ lattices. The bands correspond to interpolations (see text). The dashed line corresponds to HRG-PDG, while the solid line corresponds to HRG-QM.

continuum extrapolations will be performed in terms of the pressure and not the trace anomaly. Therefore, a more accurate determination of the pressure and the trace anomaly at low temperatures is needed. We added the following temperatures: $T=123 \mathrm{MeV}\left(N_{\tau}=10\right)$, and $T=133 \mathrm{MeV}$ and $T=140 \mathrm{MeV}\left(N_{\tau}=12\right)$. The $T=0$ gauge configurations are the same as in Ref. [8]. The numerical results for the trace anomaly in the low temperature region are shown in Fig. 3. We performed interpolations of the lattice results on $\epsilon-3 p$ using smoothing splines. The number of knots in the spline and the value of the smoothing parameter have been adjusted such that we obtain a smooth behavior with minimum number of knots and keep the $\chi^{2} / \mathrm{df}$ close to one. The statistical error on the spline has been estimated using bootstrap method. We see sizable differences in $\epsilon-3 p$ calculated with $N_{\tau}=10$ and $N_{\tau}=12$, indicating residual cutoff effects in the region $160 \mathrm{MeV}<T<180 \mathrm{MeV}$. We also compare our results with the hadron resonance gas (HRG) model. We show two versions of the HRG model: one that takes into account all states from the particle data group, which we label as HRGPDG, and one that includes baryon states that are not yet discovered experimentally, but predicted by the quark model (missing states). We label the latter model as HRG-QM. The details of the HRG models are described in Appendix B. There we also introduce the HRG-QM models for nonzero lattice spacing in addition to the continuum HRG-QM model shown in Fig. 3. From the figure we see that the difference between the two HRG models is only significant for $T>150 \mathrm{MeV}$. The lattice results for $N_{\tau}=10$ and 12 agree with the HRG models only for $T<145 \mathrm{MeV}$. This is in agreement with the previous results [7,8]. Unlike in Ref. [8] we did not require that the interpolations agree with the HRG model at low temperatures. So these results serve as independent check for the validity of the HRG model. 


\section{THE PRESSURE OF 2 + 1 FLAVOR QCD FROM LOW TO HIGH TEMPERATURES}

In this section we discuss the calculation of the pressure in the wide temperature range from $T=120 \mathrm{MeV}$ to $2000 \mathrm{MeV}$. For this purpose we combine the published HotQCD results for the trace anomaly with the results obtained for $m_{l}=m_{s} / 5$ and discussed in the previous section. We use the published HotQCD results for $T \leq$ $407 \mathrm{MeV}$ and $N_{\tau}=12, T \leq 489 \mathrm{MeV}$ and $N_{\tau}=10, T \leq$ $611 \mathrm{MeV}$ and $N_{\tau}=8$, and $T \leq 815 \mathrm{MeV}$ and $N_{\tau}=6$ [8]. For temperatures higher than these we use the new $m_{s} / 5$ results with $N_{\tau}=12,10,8$ and 6 . Since the quark mass effects are smaller than the statistical errors we treat these two data sets as one and perform interpolations of the data from the combined set. Using the resulting interpolating function we can calculate the pressure according to Eq. (2). Essentially we will be computing the pressure for lines of constant physics corresponding to $m_{l}=m_{s} / 20$ even though the data for the trace anomaly at the high temperatures come from calculations at $m_{l}=m_{s} / 5$. To fix the pressure completely we need to specify the lower integration limit $T_{0}$ as well as the value of the pressure at $T=T_{0}$. The lower integration limit $T_{0}$ is determined by the lowest data point for which a lattice calculation of $\epsilon-3 p$ is available for given $N_{\tau}$. As in Ref. [8] we will use HRG to estimate the pressure at $T_{0}$. However, when choosing the value of $p\left(T_{0}\right)$ we need to take into account the discretization effects of the staggered fermion formulation due to the distortion of the hadron spectrum. Therefore we calculate $p\left(T_{0}\right)$ in the HRG model with distorted hadron spectrum. The details of these calculations are discussed in Appendix B. As the result we obtain a value $p\left(T_{0}, N_{\tau}\right)$ for each $N_{\tau}$. The values of $T_{0}$ and $p\left(T_{0}, N_{\tau}\right)$ used in the calculation of the pressure are given in Table II.

With these inputs we can calculate the pressure for $N_{\tau}=6,8,10$ and 12. The results are shown in Fig. 4. We see significant cutoff dependence in the low temperature region and smaller cutoff dependence in the high temperature region. In the low temperature region the pressure follows qualitatively the cutoff dependence obtained in the HRG model with distorted hadron spectrum, cf. Fig. 4 (left). The continuum limit for the pressure is approached from below. The pressure shows stronger cutoff dependence than the trace anomaly. Both of these features could be

TABLE II. The values of $T_{0}$ and $p\left(T_{0}\right)$ used to calculate the pressure for different $N_{\tau}$ (see text).

\begin{tabular}{lcc}
\hline \hline$N_{\tau}$ & $T_{0}[\mathrm{MeV}]$ & $p\left(T_{0}, N_{\tau}\right)$ \\
\hline 6 & 135 & $0.189(54)$ \\
8 & 120 & $0.145(22)$ \\
10 & 125 & $0.226(23)$ \\
12 & 135 & $0.344(29)$ \\
\hline \hline
\end{tabular}

understood in the framework of the HRG model with distorted hadron spectrum (see Appendix B).

At high temperatures the cutoff dependence of the pressure can be understood in the weak-coupling picture. In this picture the pressure can be written as the sum of quark and gluon pressures with the latter being defined as the QCD pressure for $N_{f}=0 .{ }^{1}$ The cutoff dependence of the quark and gluon pressures has been studied in lattice perturbation theory up to order $\alpha_{s}[13,17,18]$. To a good approximation this cutoff dependence is described by the ideal gas result. The cutoff dependence of the gluon pressure is very small $(<1 \%)$ for $N_{\tau} \geq 6$ if improved gauge action is used [12]. This is confirmed by direct lattice numerical study [17]. Therefore we neglect it here. The cutoff dependence of the quark pressure was studied in Refs. $[13,18]$ for improved staggered actions, namely the Naik action and p4 action. The cutoff dependence of the quark pressure is much bigger than of the gluon pressure for $N_{\tau} \leq 12$. At tree level the HISQ action has the same cutoff dependence as the Naik action. Thus, the ideal gas limit for the HISQ action is determined by the result for Ref. [13]. The ideal gas limit for each $N_{\tau}$ is shown in Fig. 4 as a horizontal line. Our numerical results for the pressure at high temperatures shown in Fig. 4 (right) follow the same trend in terms of cutoff dependence as the free theory result. The $N_{\tau}=12$ result appears to be an exception, though given the statistical errors the deviations from the trend is not very significant. At quantitative level the cutoff effects in the pressure are smaller than in the free field theory. This observation is in line with the cutoff dependence of the pressure in SU(3) gauge theory [11] as well as cutoff effects of quark number susceptibilities (QNS) obtained with HISQ action $[19,20]$. For comparison we also show the pressure obtained with $\mathrm{p} 4$ action and $N_{\tau}=6$ or 8 lattices $[3,4]$. The corresponding results are significantly larger than the ones obtained with HISQ action at high temperatures and significantly lower at small temperatures. This is most likely due to the large cutoff effects related to tastesymmetry breaking for the $\mathrm{p} 4$ action (see discussions in Appendix B).

The cutoff dependence of the pressure obtained with HISQ action and p4 action closely resembles the cutoff dependence of quark number susceptibilities (QNS) defined as second and fourth derivatives of the pressure with respect to quark chemical potential,

$$
\chi_{2 n}^{q}=\frac{\partial^{2 n} p\left(T, \mu_{q}\right)}{\partial \mu_{q}^{2 n}}, \quad n=1,2, \quad q=l, s .
$$

\footnotetext{
${ }^{1}$ Note that this decomposition of the pressure into the quark and gluon pressures is different from the decomposition of $\Theta^{\mu \mu}$ into $\Theta_{G}^{\mu \mu}$ and $\Theta_{F}^{\mu \mu}$. The quark pressure does not vanish for zero quark mass but $\Theta_{F}^{\mu \mu}$ does.
} 

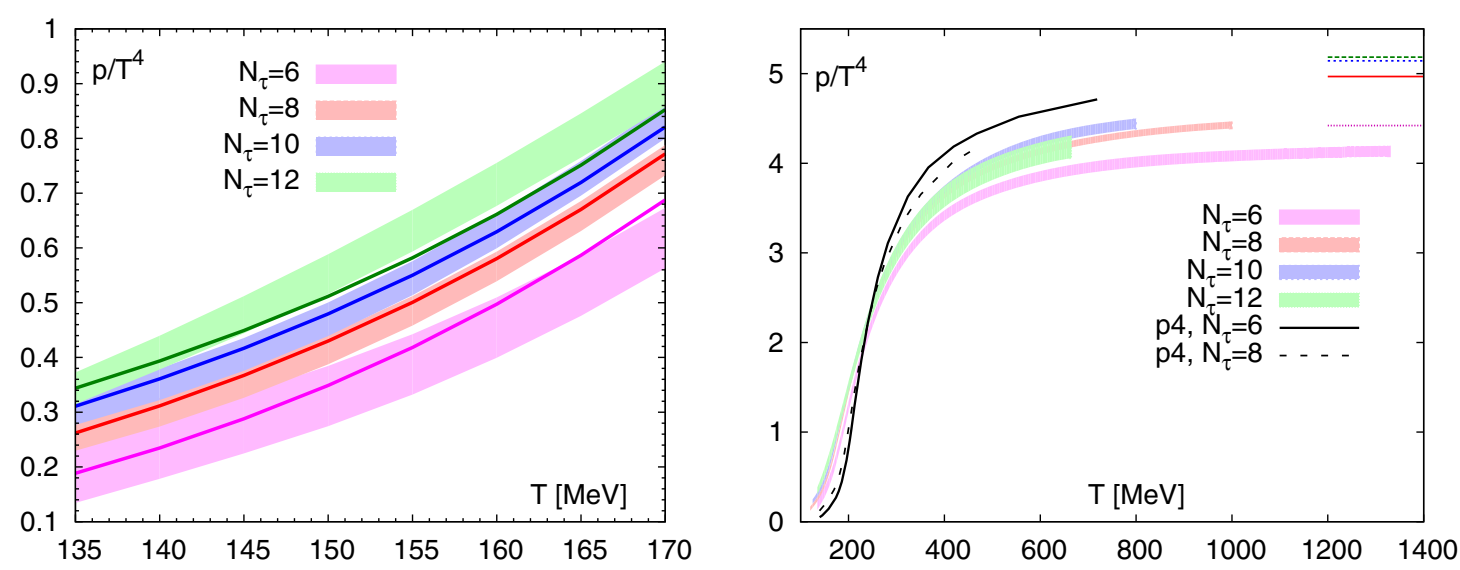

FIG. 4. Left: The pressure in the low temperature region. The lines correspond to HRG with distorted hadron spectrum (see text). Right: The pressure in the entire temperature range. The horizontal lines correspond to the free theory result. Also shown are the results for the pressure obtained with $\mathrm{p} 4$ action and $N_{\tau}=6$ or $8[3,4]$.

Since the cutoff dependence of the quark contribution to the pressure and the cutoff dependence of QNS are similar, we could use the latter to correct for the cutoff dependence of the former. This can be done as follows. We write the pressure as the sum of the quark and gluon pressures ${ }^{2}$ $p(T)=p^{q}(T)+p^{g}(T)$. Assuming that the gluonic pressure has negligible cutoff dependence (see the above discussions) we can write

$$
p(T)=p\left(T, N_{\tau}\right)+\operatorname{corr}\left(T, N_{\tau}\right),
$$

where $p\left(T, N_{\tau}\right)$ is the pressure at fixed lattice spacing $\left(N_{\tau}\right)$ and

$$
\operatorname{corr}\left(T, N_{\tau}\right)=p^{q}(T)\left(1-\frac{p^{q}\left(T, N_{\tau}\right)}{p^{q}(T)}\right)
$$

is the correction factor due to discretization errors. Here $p^{q}(T)$ stands for the quark pressure in the continuum limit, while $p^{q}\left(T, N_{\tau}\right)$ is the quark pressure at nonzero lattice spacing, $a=\left(N_{\tau} T\right)^{-1}$. If we assume that the cutoff dependence of the quark pressure is the same as of the second order QNS, $\chi_{2}^{l}$, i.e.

$$
\frac{p^{q}\left(T, N_{\tau}\right)}{p^{q}(T)} \simeq \frac{\chi_{2}^{l}\left(T, N_{\tau}\right)}{\chi_{2}^{l}(T)}
$$

we can use the results of Ref. [19] to obtain the correction provided we also have an estimate for continuum quark pressure $p^{q}(T)$. Lattice calculations show that the QCD pressure is below the ideal gas limit by about $15 \%$ at high temperatures. Therefore, the ideal quark pressure provides a fair estimate for $p_{q}(T)$. Thus, we have an estimate for the correction. We apply this correction to the pressure

\footnotetext{
${ }^{2}$ This can be done if the weak-coupling picture holds at high temperatures, as expected.
}

calculated for fixed $N_{\tau}$. The results are shown in Fig. 5 . We see from the figure that the pressure bands corresponding to different $N_{\tau}$ agree within errors, i.e. applying the corrections largely reduces the $N_{\tau}$ dependence of the results. We also see that while the $\mathrm{p} 4$ results are still higher than the HISQ results they agree within the statistical errors of the latter. The cutoff dependence of the pressure is understood because to a fairly good approximation it is given by the cutoff dependence of the free quark gas. This is not the case for the cutoff dependence trace anomaly, which would require a three-loop calculation as mentioned in Sec. I.

Now, that the cutoff dependence of the pressure is understood we can proceed with the continuum extrapolations. As discussed above at high temperatures the dominant cutoff dependence of the pressure is given by the cutoff dependence of the ideal quark gas, and therefore,

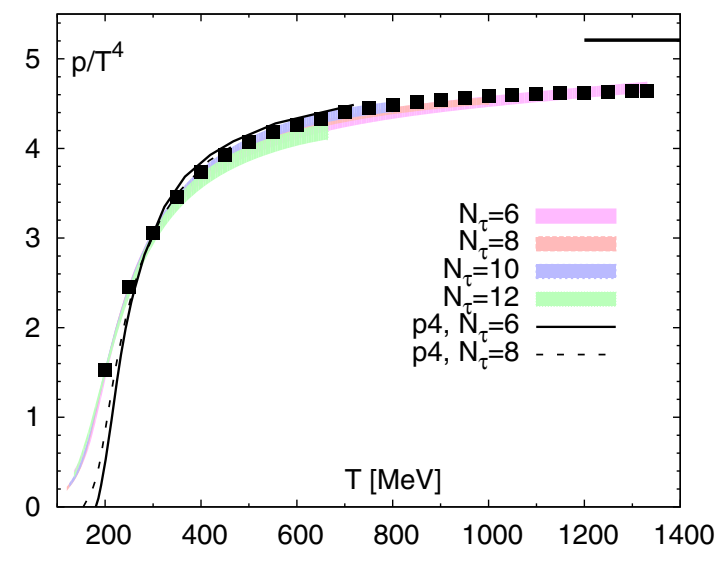

FIG. 5. The pressure calculated with HISQ action for different $N_{\tau}$ and corrected for cutoff effects. The filled squares are the continuum results for the pressure. For comparison we also plot the $\mathrm{p} 4$ results for the pressure corrected for cutoff effects at high temperatures. 
for improved staggered actions like HISQ it is expected to scale like $1 / N_{\tau}^{4}$. This expectation is confirmed by the study of QNS at high temperatures with HISQ action [19,20]. On the other hand at low temperatures the dominant cutoff effects are due to taste-symmetry breaking of staggered fermions and scale like $a^{2} \sim 1 / N_{\tau}^{2}$. This is also confirmed by lattice calculations [21]. We find that the cutoff dependence of the pressure is incompatible with $1 / N_{\tau}^{2}$ behavior for $T>400 \mathrm{MeV}$. Similar findings have been obtained for QNS $[19,20]$. On the other hand, for $T<$ $200 \mathrm{MeV}$ we find that $1 / N_{\tau}^{4}$ behavior of the cutoff effects is incompatible with the data. Therefore, we will assume that the cutoff effects are proportional to $1 / N_{\tau}^{4}$, when performing continuum extrapolations for $T>400 \mathrm{MeV}$. In the intermediate temperature region, $200 \mathrm{MeV}<T<$ $400 \mathrm{MeV}$ the cutoff effects should be proportional to some combination of $1 / N_{\tau}^{2}$ and $1 / N_{\tau}^{4}$. Thus, one should in principle fit the data by $a / N_{\tau}^{2}+b / N_{\tau}^{4}$ form to obtain the continuum limit. But because we have only four $N_{\tau}$ values and the errors of the $N_{\tau}=10$ and $N_{\tau}=12$ data are large the continuum result obtained from such fits has large statistical error. It turns out, however, that in this intermediate temperature region it is possible to fit the cutoff dependence of the pressure with $1 / N_{\tau}^{2}$ form as well as with $1 / N_{\tau}^{4}$ form and obtain $\chi^{2} / \mathrm{df} \sim 1$. The $1 / N_{\tau}^{2}$ fits give higher values of the pressure than the $1 / N_{\tau}^{4}$ fits, though the results from both fits overlap within the error bands. The difference between the central values of the $1 / N_{\tau}^{2}$ and $1 / N_{\tau}^{4}$ fits could be considered as a measure of the systematic error of the continuum extrapolation. This difference turns out to be about the same as the statistical errors of the $1 / N_{\tau}^{4}$ extrapolations. Therefore, we estimate the total error of the continuum pressure for $200 \mathrm{MeV}<T<400 \mathrm{MeV}$ by doubling the statistical error of the $1 / N_{\tau}^{4}$ fit. Alternatively we could add the systematic error estimated as described above with the statistical error in quadrature to obtain the total error. The corresponding errors will be smaller. We prefer to be more conservative. Similar analysis as above has been performed to obtain the continuum extrapolated result for the entropy density.

In the temperature interval $200 \mathrm{MeV}<T<660 \mathrm{MeV}$ we have four lattice spacings to perform continuum extrapolations. As the result the continuum extrapolations are most reliable in this temperature interval. In the temperature range $660 \mathrm{MeV}<T<800 \mathrm{MeV}$ we have three lattice spacings, so controlled continuum extrapolation is still possible. For $T>800 \mathrm{MeV}$, we can only provide a continuum estimate for the pressure. For $T<$ $1000 \mathrm{MeV}$ we do this by performing const $+1 / N_{\tau}^{4}$ fit of the $N_{\tau}=6$ and 8 data for the pressure. Interestingly, it turns out that the coefficient of $1 / N_{\tau}^{4}$ obtained when using only $N_{\tau}=6$ and 8 data, and when using $N_{\tau}=6,8$ and 10 data is the same within errors for $T \simeq 800 \mathrm{MeV}$. So perhaps this extrapolation is not totally out of control. Finally, to obtain the continuum estimate for $T>1000 \mathrm{MeV}$ we assume that the coefficient of the $1 / N_{\tau}^{4}$ term is the same as obtained from the fit of the $N_{\tau}=6$ and 8 data at $T=995 \mathrm{MeV}$ and correct the $N_{\tau}=6$ pressure by $1 / 6^{4}$ times this coefficient. The continuum results for the pressure obtained using this procedure are shown in Fig. 5 and compared to the corrected results for $N_{\tau}=6,8,10$ and 12 . The continuum result for the pressure agrees with the corrected results. This serves as an important cross-check for our continuum extrapolations for $T<1330 \mathrm{MeV}$.

For comparison of the lattice calculation of the EoS with the weak-coupling results it is important to have an alternative method to obtain continuum results for $T>$ $800 \mathrm{MeV}$ and also extend the calculation to higher temperatures. In order to do this we consider again the trace anomaly. As discussed in Sec. III for $N_{\tau} \geq 8$ and $T>$ $300 \mathrm{MeV}$ we do not see any cutoff dependence of the trace anomaly. This also means that given the statistical errors the $N_{\tau}=8$ results for the trace anomaly can be considered as the continuum ones. Therefore, we can perform a combined interpolation of the numerical results for the trace anomaly obtained with $N_{\tau}=8,10$ and 12 in the temperature interval $300 \mathrm{MeV}<T<1000 \mathrm{MeV}$, providing a continuum estimate. The $N_{\tau}=4$ and 6 results for the trace anomaly lie below this continuum estimate. However, if we rescale the $N_{\tau}=4$ and 6 results on the trace anomaly by factors 1.2 and 1.4 , respectively, they agree with the above continuum estimate for $800 \mathrm{MeV}<T<1000 \mathrm{MeV}$ within errors. This is demonstrated in Fig. 6. Therefore, to obtain a continuum estimate for the trace anomaly beyond $T=$ $1000 \mathrm{MeV}$ we re-scale the $N_{\tau}=4$ and $N_{\tau}=6$ data for $T>1000 \mathrm{MeV}$ with the above factors. Here we tacitly assume that the cutoff dependence of the trace anomaly is temperature independent. This assumption, however, is quite reasonable since the cutoff dependence at high temperatures should be described by weak-coupling

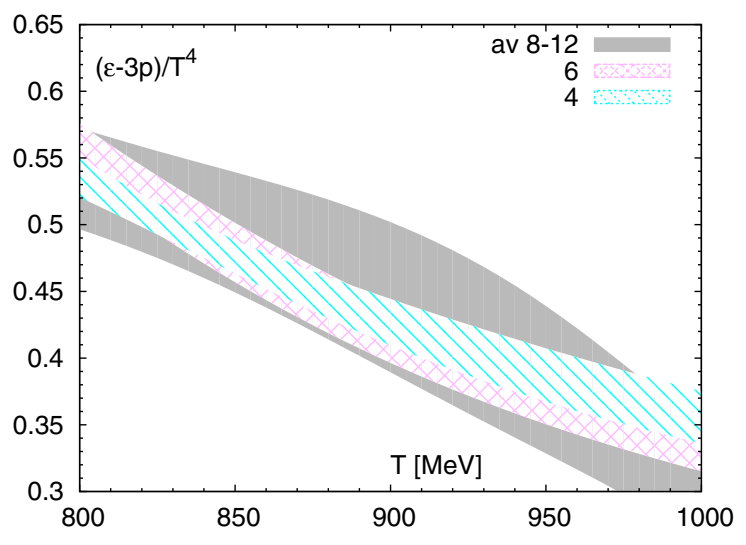

FIG. 6. The interpolation of the lattice results for $(\epsilon-3 p) / T^{4}$ for $N_{\tau}=8,10$ and 12 compared with the interpolations for $(\epsilon-3 p) / T^{4}$ obtained with $N_{\tau}=4$ and 6 . The latter have been multiplied by 1.2 and 1.4 to bring them into agreement with the former interpolation. 
expansion and thus is proportional to $a^{2}=1 /\left(N_{\tau} T\right)^{2}$ times the coupling constant to some power. Since the coupling constant depends on the temperature scale logarithmically in a limited temperature interval the cutoff effects should be approximately temperature independent. Our study of the $N_{\tau}$ dependence of the pressure for $T>400 \mathrm{MeV}$ confirms this expectation. The cutoff dependence of the quark number susceptibilities $[19,20]$ and the free energy of the static quark [15] also support this assumption. Therefore, we perform a spline interpolation of the combined $N_{\tau}=12,10,8,6$ and 4 data in the temperature interval $400 \mathrm{MeV}<T<2000 \mathrm{MeV}$. Because we corrected the trace anomaly obtained on $N_{\tau}=4$ and $N_{\tau}=$ 6 lattices we assign an additional systematic error of $20 \%$ and $40 \%$ to the corresponding data points before the interpolation, i.e. the size of the systematic errors that we assume is the same as the magnitude of the correction. Using this interpolation we calculate the integral of the trace anomaly from $T=660 \mathrm{MeV}$ to $2000 \mathrm{MeV}$, which together with the continuum result for the pressure at $660 \mathrm{MeV}$ obtained above gives us the continuum pressure estimate that extends to temperatures as high as $2000 \mathrm{MeV}$. From the pressure we can also calculate the entropy density. These calculations will be used in the next section for the comparison with the weak-coupling results. We also compared this continuum estimate of the pressure with the one discussed before. For $T<1330 \mathrm{MeV}$ we find excellent agreement between the two continuum estimates.

We note that our continuum result for $T=500 \mathrm{MeV}$ is one and a half sigma higher than the continuum result of Ref. [7], while our continuum estimate for higher temperatures is $5-7 \%$ higher than the continuum estimate of Ref. [6]. Our continuum result for the pressure for $T<$ $400 \mathrm{MeV}$ agrees very well with the HotQCD result [8] but has considerably smaller errors.

\section{EQUATION OF STATE AT HIGH TEMPERATURES AND COMPARISON WITH WEAK-COUPLING CALCULATIONS}

In this section we compare the lattice results on the EoS with the weak-coupling calculations. We start our discussion with the trace anomaly. In Fig. 7 we compare our lattice results for the trace anomaly obtained with $N_{\tau}=8$, 10 and 12 as well as the corrected results for $N_{\tau}=4$ and 6 (see previous section) with the results of three-loop HTL perturbation theory [22]. We see good agreement between the lattice results and the results obtained in three-loop HTL perturbation theory, although the error band of the latter is still quite large. The lattice results on the trace anomaly agree very well with the weak-coupling calculations based on dimensionally reduced effective field theory, the electrostatic QCD (EQCD) [10].

Next we compare the high temperature lattice results for the pressure and the entropy density with the three-loop HTL perturbation theory [22] and the results obtained using

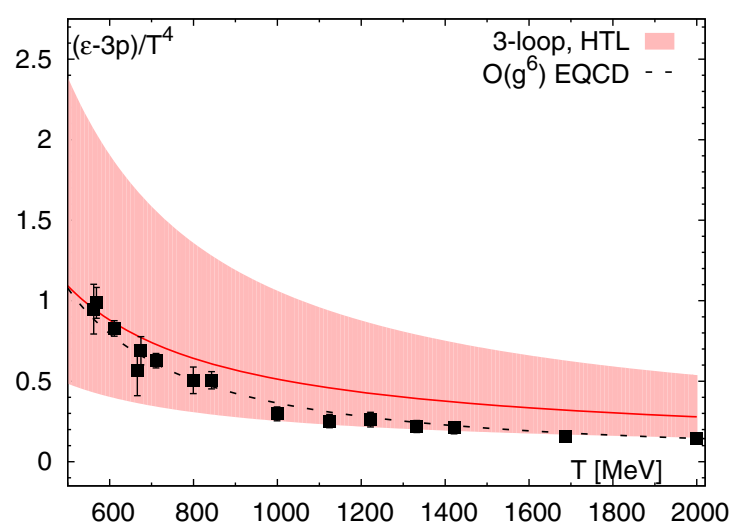

FIG. 7. The comparison of the lattice results for the trace anomaly with three-loop HTL perturbation theory shown as the line and the band. The size of the band corresponds to the scale variation from $\mu=\pi T$ to $4 \pi T$. Also shown as a dashed line is the EQCD result for the trace anomaly (see text). The lattice results for $N_{\tau}=4$ and 6 for $T>600 \mathrm{MeV}$ have been scaled by 1.2 and 1.4 , respectively.

EQCD [10]. The comparison is shown in Fig. 8. For $T<$ $660 \mathrm{MeV}$ we use the continuum extrapolated lattice results obtained from the calculations on $N_{\tau}=6,8,10$ and 12 lattices. For higher temperatures, we use the continuum estimate based on the trace anomaly calculated on the coarsest $N_{\tau}=4$ lattice. As discussed in the previous section this continuum estimate is validated by direct continuum extrapolation for $T<1000 \mathrm{MeV}$, but at higher temperatures it relies on the temperature independence of the cutoff effects. Therefore, in Fig. 8 we show the continuum estimate as open symbols. We see that the EQCD calculations are higher than our lattice results. Our lattice results lie above the central value of the three-loop HTL perturbative result by one sigma. However, the lattice data are fully contained within the uncertainty of the threeloop HTL result. In the considered temperature range the uncertainty of the lattice results is significantly smaller than the uncertainty of the three-loop HTL result. For the entropy density we also compare our lattice results with the resummed perturbative calculations in next-to-leading log approximation (NLA) [23]. This comparison is shown in Fig. 8 (right). The NLA calculation leads to higher entropy density than the lattice result, although overlaps within the uncertainty with latter for $T>1300 \mathrm{MeV}$. The NLA calculation is based on the $\Phi$ derivable approach [2426]. In this approach one calculates the derivatives of the pressure, which leads to cancellation of many higher order diagrams. As the result one obtains relatively simple expressions for the entropy density [26] or the quark number susceptibility [27]. The calculation of the pressure in this approach, however, is difficult.

It is clear that our lattice results are sufficiently precise to test the various weak-coupling approaches and it would be desirable to further reduce the uncertainty of the 

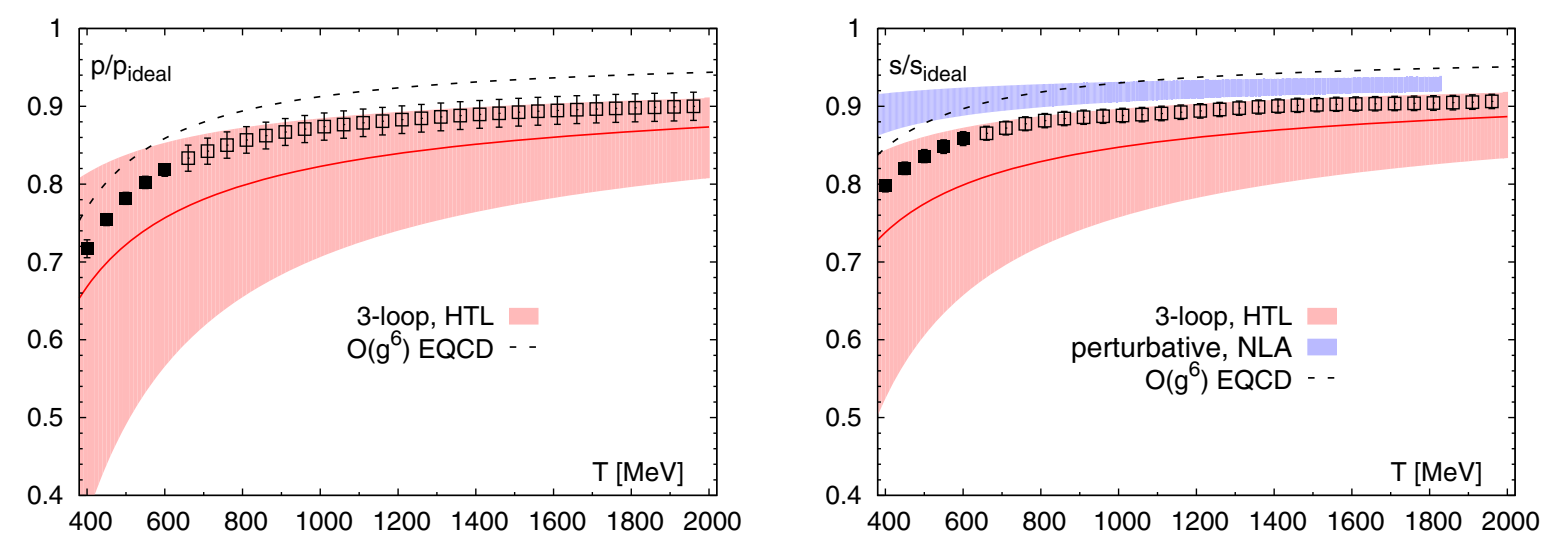

FIG. 8. The pressure (left) and the entropy density (right) in the high temperature region compared with the weak-coupling calculations. The filled symbols correspond to the continuum results obtained from lattice calculations on $N_{\tau}=6,8,10$ and 12 lattices. The open symbols correspond to continuum estimate (see text). The errors of the continuum estimate have been enlarged by factor two to indicate additional systematic errors that might be present. The red line and the band correspond to the three-loop HTL perturbation theory [22], the blue band corresponds to the resummed calculation in next-to-leading log approximation (NLA) [23]. The width of the bands correspond to the scale variation from $\mu=\pi T$ to $4 \pi T$. Also shown is the weak-coupling result obtained in EQCD [10].

weak-coupling approaches to see if the thermodynamics of the quark gluon plasma can be indeed understood using the weak-coupling expansion in the considered temperature range.

\section{CONCLUSIONS}

We extended the previous calculation of the EoS with HISQ action to higher temperatures. First, we extended the calculation of the trace anomaly to higher temperatures using lattice simulations at larger quark mass, $m_{l}=m_{s} / 5$. We showed that the quark mass dependence of the trace anomaly is negligible for $T>400 \mathrm{MeV}$ given the statistical error. Then using the results on the trace anomaly and the integral method we calculated the pressure for $N_{\tau}=6,8,10$ and 12 . We studied the cutoff $\left(N_{\tau}\right)$ dependence of the pressure and performed the continuum extrapolation in the high temperature limit. We pointed out that the cutoff dependence of the pressure is dominated by the quark contribution and the cutoff dependence of this contribution is very similar to the cutoff dependence of QNS at high temperatures. We also showed that using the known cutoff dependence of QNS it is possible to correct for the cutoff effects in the pressure at fixed $N_{\tau}$. The corrected results for the pressure calculated with HISQ action and $\mathrm{p} 4$ action for different $N_{\tau}$ agree within errors and also agree with the continuum result. Thus, we achieved a controlled continuum extrapolation of the pressure at high temperatures. Finally, using $N_{\tau}=4$ and 6 results on the trace anomaly we provided a continuum estimate for the pressure that extends to temperatures as high as $T=2000 \mathrm{MeV}$. We compared this continuum estimate with the weak-coupling calculations and found a reasonably good agreement between the lattice and the weak-coupling results.

\section{ACKNOWLEDGMENTS}

The simulations have been carried out on the computing facilities of the Computational Center for Particle and Astrophysics (C2PAP), SuperMUC and NERSC. We used the publicly available MILC code to perform the numerical simulations [28]. The data analysis was performed using the R statistical package [29]. We thank F. Karsch for providing the numerical values of the free quark pressure for finite $N_{\tau}$. We also thank M. Strickland and N. Haque for sending the 3-loop HTL results for the EoS. This work has been supported in part by the U.S. Department of Energy through grant Contract No. DE-SC0012704. J. H. Weber acknowledges the support by the Bundesministerium für Bildung und Forschung (BMBF) under Grant No. "Verbundprojekt 05P2015-ALICE at High Rate (BMBF-FSP 202) GEMTPC Upgrade and Field theory based investigations of ALICE physics" under Grant No. 05P15WOCA1.

\section{APPENDIX A: ZERO TEMPERATURE CALCULATIONS}

For $\beta=7.03$ and 7.825 we generated a single stream of $\mathrm{MC}$ evolution. For the highest three $\beta$ values we generated three streams of MC evolution called $a, b$ and c; each of these streams corresponds to a single value of topological charge $Q$. The lengths of these streams for every value of $\beta$ are 1389, 1269 and 1269, respectively. The values of the plaquette, rectangles, light and strange quark condensates are given in Table III together with the values of $Q$. The values of rectangles and plaquettes are the same within errors for the streams with different $Q$. For the quark condensate we see small, but in some cases statistically significant differences. The difference in the value of the light quark condensate is between $1 \%$ and $2 \%$, while for the 
TABLE III. The values of plaquette, rectangle, light and strange quark condensates at $T=0$ for different topological sectors $Q=0,1,2$.

\begin{tabular}{|c|c|c|c|c|c|c|}
\hline$\beta$ & plaquette & $\langle\bar{\psi} \psi\rangle_{l}$ & $\langle\bar{\psi} \psi\rangle_{s}$ & rectangle & Q & stream \\
\hline \multirow{4}{*}{8.0} & $0.6641244(21)$ & $0.0026400(66)$ & $0.0117787(51)$ & $0.4607658(29)$ & 2 & $\mathrm{a}$ \\
\hline & $0.6641256(15)$ & $0.0026061(122)$ & $0.0117719(76)$ & $0.4607666(23)$ & 1 & $\mathrm{~b}$ \\
\hline & $0.6641257(24)$ & $0.0025923(87)$ & $0.0117889(59)$ & $0.4607666(35)$ & 0 & $\mathrm{c}$ \\
\hline & $0.6738855(16)$ & $0.00207849(52)$ & $0.0095507(55)$ & $0.4744956(24)$ & 2 & $\mathrm{a}$ \\
\hline \multirow[t]{3}{*}{8.2} & $0.6738854(12)$ & $0.00199916(69)$ & $0.0095271(60)$ & $0.4744943(17)$ & 0 & $\mathrm{~b}$ \\
\hline & $0.6738865(12)$ & $0.00201003(95)$ & $0.0095399(75)$ & $0.4744971(18)$ & 0 & $\mathrm{c}$ \\
\hline & $0.6830217(14)$ & $0.00171386(47)$ & $0.0078134(48)$ & $0.4874515(22)$ & 2 & $\mathrm{a}$ \\
\hline \multirow[t]{2}{*}{8.4} & $0.6830200(17)$ & $0.00158675(57)$ & $0.0077629(71)$ & $0.4874514(28)$ & 0 & $\mathrm{~b}$ \\
\hline & $0.6830187(12)$ & $0.00161808(63)$ & $0.0077963(54)$ & $0.4874474(18)$ & 0 & $\mathrm{c}$ \\
\hline
\end{tabular}

strange quark condensates it is $<0.5 \%$. For $\beta=8.4$ there are differences in the values of the light quark condensate also for the streams that belong to the same topological sector, which appear to be statistically significant. This is most likely due to the fact that each of the streams is relatively short. These small differences, are taken into account in the calculations as additional systematic errors. However, the additional systematic effects are largely irrelevant for the calculation of the trace anomaly, since the highest three beta values correspond to temperatures $T>400 \mathrm{MeV}$, where the contribution of quark condensates is very small.

To determine the lattice spacing we calculated the static quark antiquark potential. We followed the same procedure as in Ref. [8], in particular the same fit ranges in time were used. For the highest three $\beta$ values we used a fit range in $t / a$, which is about the same as in Ref. [8] for $\beta=7.825$. Our results for the potential are shown in Fig. 9. We calculated the static quark antiquark potential for different topological sectors and did not see any dependence on the topological charge within the statistical errors.

It is interesting to compare the potential calculated for $m_{l}=m_{s} / 5$ and $m_{s} / 20$ at the same value of $\beta$. Such a comparison is shown in Fig. 10 for $\beta=7.03$. As one can see from the figure the quark mass effects are very small for

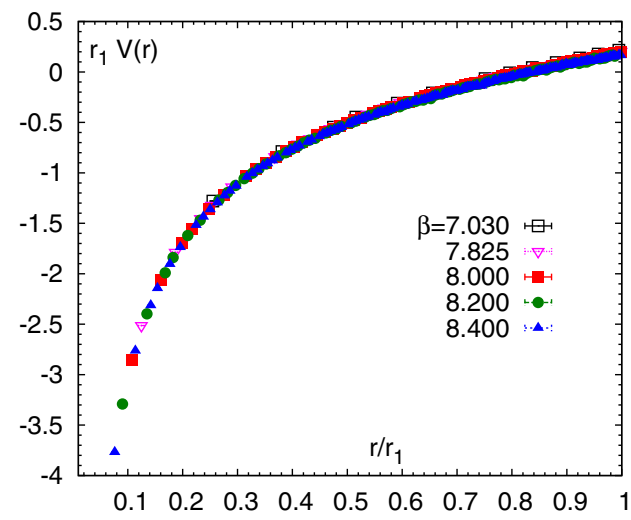

FIG. 9. The potentials calculated for $m_{l}=m_{s} / 5$ at $\beta=7.030$, $7.825,8.00,8.20$ and 8.40 . $r<r_{1}$. They are the smallest at the shortest distance and gradually increase with increasing $r$. However, even for $r=r_{1}$ the effects are smaller than $0.2 \%$ and for $r<0.8 r_{1}$ are smaller than $0.1 \%$. We get very similar results for $\beta=7.825$.

We also find that the difference between the potential calculated for $m_{s} / 5$ and $m_{s} / 20$ in units of $r_{1}$ can be parametrized as

$$
\Delta V(r)=V^{m_{s} / 5}(r)-V^{m_{s} / 20}(r)=b\left(r / r_{1}\right)^{2} .
$$

We get $b=0.00577(19)$ for $\beta=7.03$ and $b=$ $0.00774(51)$ for $\beta=7.825$. From these we can estimate that $r^{2} V^{\prime \prime}(r)$ is changed by about $1.4 \%$ around $r=r_{1}$, and by about $0.2 \%$ or less around $r=r_{2}$, when changing $m_{l}$ from $m_{s} / 20$ to $m_{s} / 5$. Therefore, we expect shifts in the values of $r_{1}$ and $r_{2}$ with changing quark masses, which are similar in magnitude.

The $r_{1}$ scale obtained from the potential at $\beta=7.03$, $7.825,8.00,8.20$ and 8.40 is given in Table IV. For $\beta \geq$ 7.825 we also calculated the $r_{2}$ scale. Moreover, this scale was calculated for $m_{l}=m_{s} / 20$ using the data on the potential from [8]. The results are given in Tab. IV. We see that the $r_{1}$ scale is about $1 \%$ smaller for $m_{l}=m_{s} / 5$ than for $m_{s} / 20$. This difference is consistent with the above

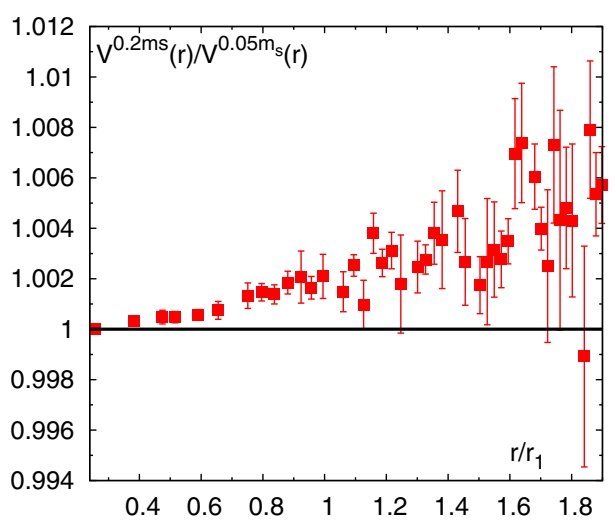

FIG. 10. The ratio of the potentials calculated for $m_{l}=m_{s} / 20$ and $m_{l}=m_{s} / 5$ for $\beta=7.03$. 
TABLE IV. The value of the scale parameters for different $\beta$ values and quark masses used in this study.

\begin{tabular}{lclc}
\hline \hline$\beta$ & $m_{l} / m_{s}$ & \multicolumn{1}{c}{$r_{1}$} & $r_{2}$ \\
\hline 8.400 & $1 / 5$ & $12.560(130)$ & $5.742(31)$ \\
8.200 & $1 / 5$ & $10.653(60)$ & $4.861(36)$ \\
8.000 & $1 / 5$ & $8.905(60)$ & $4.075(30)$ \\
7.825 & $1 / 5$ & $7.570(104)$ & $3.469(18)$ \\
7.825 & $1 / 20$ & $7.690(58)$ & $3.479(21)$ \\
7.596 & $1 / 20$ & $6.336(56)$ & $2.865(11)$ \\
7.373 & $1 / 20$ & $5.172(34)$ & $2.350(40)$ \\
7.030 & $1 / 5$ & $3.737(13)$ & - \\
7.030 & $1 / 20$ & $3.763(13)$ & - \\
\hline \hline
\end{tabular}

expectations and statistically it is not very significant. The value of $r_{2}$ at $\beta=7.825$ is $0.3 \%$ smaller for $m_{l}=m_{s} / 5$ than for $m_{l}=m_{s} / 20$. Again this difference is statistically not significant. Since the $r_{2}$ scale shows smaller quark mass dependence we could use it to extend the scale setting procedure of Ref. [8] to higher $\beta$, namely up to $\beta=8.40$. To do this we first consider the ratio $r_{2} / r_{1}$, which is shown in Fig. 11. We do not see any $\beta$ dependence of this ratio within errors. Fitting $\beta>7.825$ we get $2.188(12)$, while the fit with $\beta \geq 7.825$ we obtain 2.210 (14). Finally the fits for all $\beta$ in the interval $[7.373: 8.400]$ we get

$$
\left(\frac{r_{1}}{r_{2}}\right)_{a v}=2.198 \pm 0.009
$$

which agrees with the above values within errors. Since $r_{2}$ is essentially mass independent and more accurately determined than $r_{1}$ for the highest $\beta$ values we will use it for the scale setting. We combine the results of Ref. [8] together with $\left(r_{1} / r_{2}\right)_{a v} \cdot r_{2}$ for $\beta=7.596,7.825,8.000$, 8.200 and 8.400 from Table IV to obtain the lattice spacing in units of $r_{1}$ in the $\beta$ region that extends to $\beta=8.400$. As in Ref. [8] we fit $a / r_{1}$ with an Allton-type form [30]:

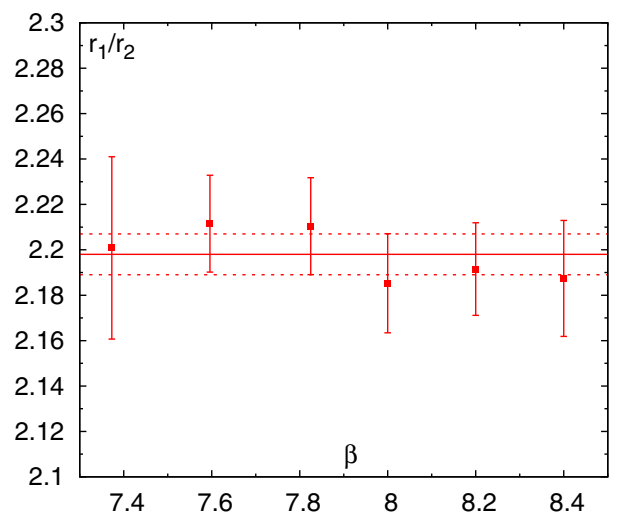

FIG. 11. The ratio of the ratio of scales $r_{1}$ and $r_{2}$ as function of $\beta$. Also shown is the fit (solid line) and its uncertainty (dashed lines).

$$
\begin{gathered}
\frac{a}{r_{1}}=\frac{c_{0} f(\beta)+c_{2}(10 / \beta) f^{3}(\beta)}{1+d_{2}(10 / \beta) f^{2}(\beta)}, \\
f(\beta)=\left(\frac{10 b_{0}}{\beta}\right)^{-\frac{b_{1}}{2 b_{0}^{2}}} \exp \left(-\frac{\beta}{20 b_{0}}\right) .
\end{gathered}
$$

Here $b_{0}$ and $b_{1}$ are the well-known coefficients of the twoloop beta function, which for the three-flavor case read $b_{0}=9 /\left(16 \pi^{2}\right), b_{1}=1 /\left(4 \pi^{4}\right)$. Fitting the combined data set for the coefficients $c_{0}, c_{2}$ and $d_{2}$ we get:

$$
\begin{gathered}
c_{0}=43.12 \pm 0.18, \\
c_{2}=347008 \pm 32131, \\
d_{2}=5584 \pm 599, \\
\chi^{2} / \mathrm{df}=0.25 .
\end{gathered}
$$

The above errors have been estimated by bootstrap method and they are smaller than those in [8], in particular the error on $c_{0}$ is reduced from 0.3 to 0.18 . This fit is shown in Fig. 12 with the band indicating its uncertainty. The difference between this parametrization of $a / r_{1}$ and the one in Ref. [8] is less than $0.3 \%$ in the entire range of $\beta$. It is interesting to note that for the highest $\beta$ value the deviation from the asymptotic 2-loop result is only one sigma. From this fit we can determine the smoothed value of $r_{1} / a$ for each value of $\beta$ and thus the temperature scale.

For the calculation of the EoS we also need the nonperturbative beta function defined as

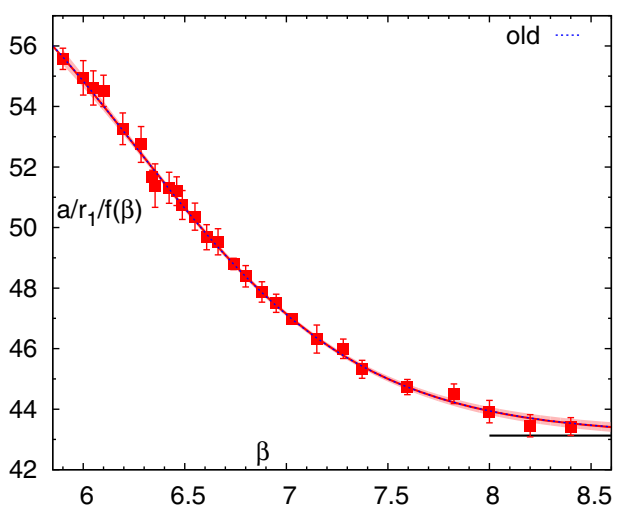

FIG. 12. The lattice spacing in units of $r_{1}$ as function of $\beta$. For better visibility we divided $a / r_{1}$ by the 2-loop beta function given by Eq. (A4). The horizontal line shows the asymptotic 2-loop result. Also shown as a dashed line is the old parametrization of $a / r_{1}$ from Ref. [8]. 


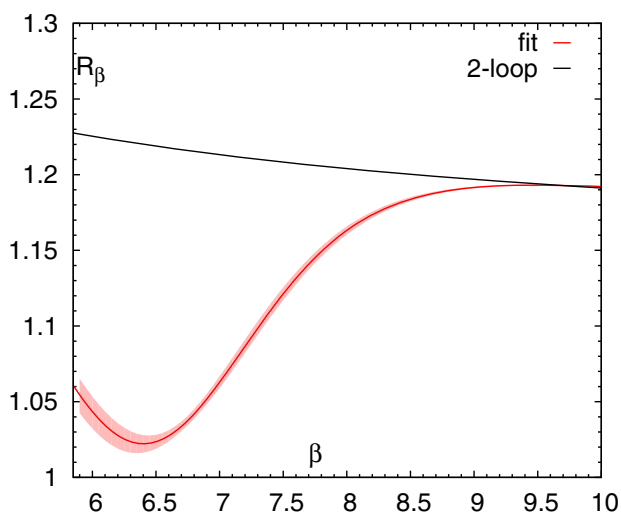

FIG. 13. The logarithmic derivative of the nonperturbative beta function.

$$
R_{\beta}=-a \frac{d \beta}{d a}=\frac{r_{1}}{a}\left(\frac{d\left(r_{1} / a\right)}{d \beta}\right)^{-1} .
$$

This is shown in Fig. 13. We also calculated the mass of the unmixed $\eta_{s s}$ meson for $\beta=8.0$ and obtained $a m_{\eta_{s s}}=0.12282(40)$. This value agrees well with determination of $m_{s}^{L C P}(\beta)$ given in Ref. [8]. For $\beta=8.2$ and 8.4 the lattices are too small to determine $a m_{\eta_{s s}}$ reliably.

\section{APPENDIX B: THE HADRON RESONANCE GAS AND CUTOFF EFFECTS AT LOW TEMPERATURES}

In the HRG model the partition function of strongly interacting matter at low temperatures is given by the partition function of noninteracting hadrons and resonances

$$
\begin{aligned}
p^{H R G} / T^{4}= & \frac{1}{V T^{3}} \sum_{i \in \text { mesons }} \ln \mathcal{Z}^{M}\left(m_{i}, T, V\right) \\
& +\frac{1}{V T^{3}} \sum_{i \in \text { baryons }} \ln \mathcal{Z}^{B}\left(m_{i}, T, V\right),
\end{aligned}
$$

where

$\ln \mathcal{Z}^{M / B}\left(m_{i}, T, V\right)=\mp \frac{V d_{i}}{2 \pi^{2}} \int_{0}^{\infty} d k k^{2} \ln \left(1 \mp e^{-E_{i} / T}\right)$,

with energies $E_{i}=\sqrt{k^{2}+m_{i}^{2}}$ and degeneracy factors $d_{i}$. The superscripts $M$ and $B$ refer to mesons and baryons. Usually the sum in the above equation contains all the meson and baryons from the Particle Data Group (PDG). However, our information of the baryon spectrum may be incomplete. There are lots of baryon states predicted by the quark model (QM) [31] as well as by lattice QCD [32] that are not included in the PDG. These are the so-called missing states. It was shown that these missing states are important for QCD thermodynamics [33-37]. Therefore, we included these missing states in the HRG model. We used the baryon spectrum from the quark model calculations of Refs. [38,39]. We call this model HRGQM. For the strange baryons we also used the spectrum from Ref. [31] and found that this only results in very small differences relative to the above calculation. The difference between the HRG-QM and the HRG model which includes only hadrons from PDG, and therefore is called HRG-PDG, is visible only for $T>150 \mathrm{MeV}$. At these temperatures, however, the HRG model itself may not be reliable.

The hadron resonance gas can be used as a tool to understand the cutoff effects in the EoS at low temperatures. Lattice discretization errors will modify the hadron spectrum which then leads to the modification of the HRG. This has been discussed in some details for $\mathrm{p} 4$ and asqtad actions [40]. Below we will discuss the discretization effects in the hadron spectrum for the HISQ action and their effect on thermodynamics of hadrons.

The staggered fermion formulation describes four flavors (tastes) of quarks in the continuum limit. To describe a single quark flavor one takes the fourth root of the staggered fermion determinant in the path integral of QCD. This is the so-called rooting trick and amounts to averaging over the four staggered tastes for each physical flavor. For the discussion of the cutoff effects on the hadron spectrum we first limit ourselves to the original four-flavor case. There are 16 pseudo-scalar (ps) mesons, which are the Goldstone bosons of the theory. At nonzero lattice spacing only a $U(1)$ subgroup of the $S U(4)_{A}$ group is preserved, and there is only one Goldstone boson in the chiral limit. The other ps mesons have squared masses proportional to $a^{2}, \delta m_{\mathrm{ps}_{i}} e_{i} a^{2}$. The breaking of the full chiral symmetry to a $U(1)$ subgroup and the corresponding splitting of ps mesons is referred to as taste-symmetry breaking. It is the largest source of discretization errors in today's lattice calculations with staggered fermions. The size of tastesymmetry breaking, i.e. the value of coefficients $e_{i}$ can be reduced by using improved actions. All improved staggered actions (p4, asqtad, stout and HISQ) reduce the size of taste-symmetry breaking to some degree, The HISQ action has the smallest taste-symmetry breaking among the improved staggered fermion actions [41]. The taste symmetry breaking effects are particularly large for the p4 action.

Taste-symmetry breaking also causes nondegeneracy of vector mesons and baryons that belong to different tastes. However, the corresponding mass splittings are much smaller than in the case of ps mesons. For the HISQ action they are of the size of statistical errors and therefore can be neglected in the following discussion. The dominant effects of taste-symmetry breaking in the vector meson and baryon sectors come from the fact that the calculations are effectively performed at larger value of the pion mass than the physical one if the lattice spacing is nonzero. Since hadronic quantities like hadron masses and decay constants decrease with decreasing pion masses, we expect that the continuum limit for these quantities is approached from 

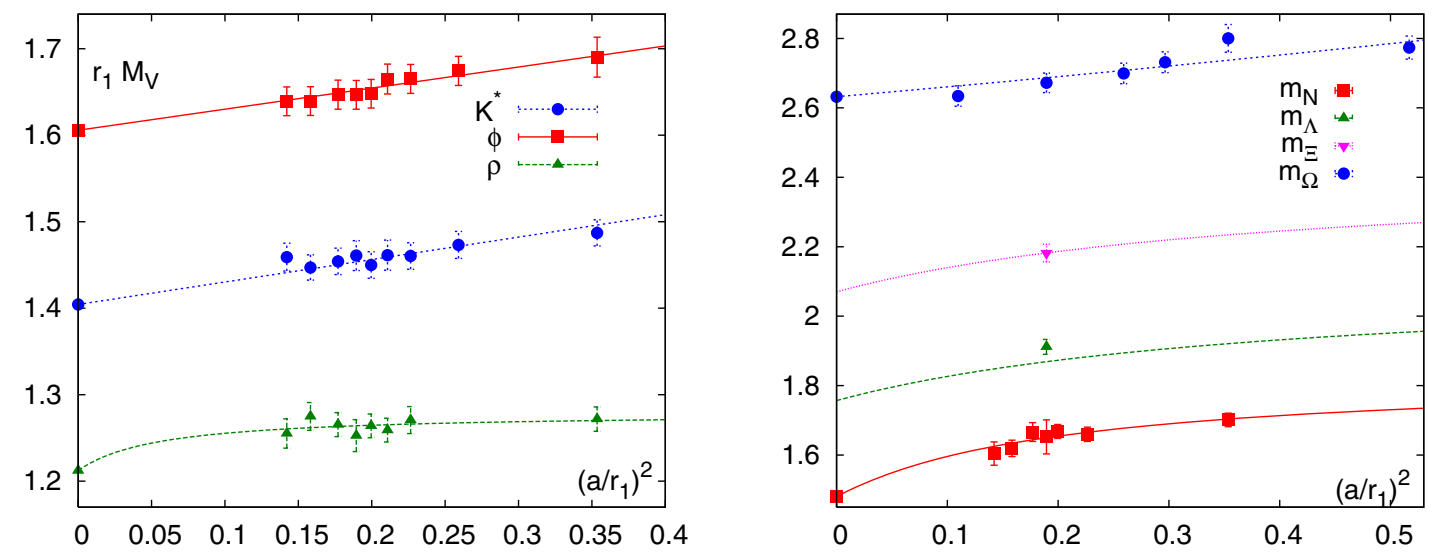

FIG. 14. The lattice spacing dependence of vector meson (left) and baryon masses (right). The lines show fits using Eq. (B3).

above. The masses of the vector mesons, nucleons and $\Omega$ baryons have been calculated with HISQ action for different lattice spacings $[8,41]$. We complement these studies by also calculating the masses of octet baryons with strangeness $S=-1$ and $S=-2$ for $\beta=10 / g^{2}=6.515$ corresponding to lattice spacing $a=0.135 \mathrm{fm}$. In Fig. 14 we show the vector meson and baryon masses as function of the lattice spacing. We see that following our expectations the hadron masses approach their continuum limit from above. We fit the $a$-dependence of the hadron masses by the form

$$
r_{1} m_{H}=\left(r_{1} m_{H}\right)^{\mathrm{cont}}+\frac{b_{H}\left(a / r_{1}\right)^{2}}{1+c_{H}\left(a / r_{1}\right)^{2}}
$$

The values of $b_{H}$ and $c_{H}$ are given in Table V. The resulting fits are also shown in Fig. 14 as lines and describe the data fairly well. For $S=-1$ and $S=-2$ baryons we could not perform the above fits. We model their lattice spacing dependence using Eq. (B3) with coefficients $b_{H}$ and $c_{H}$ obtained for the nucleon and divided by two and three, respectively. This seems to capture the cutoff effects in $S=-1$ and $S=-2$, see Fig. 14 .

To reduce cutoff effects in the thermodynamic quantities it has been suggested to use the kaon decay constant $f_{K}$ to set the lattice spacing. Since $f_{K}$ shows an $a$-dependence that is similar to that of the hadron masses the ratios $m_{H} / f_{K}$ are expected to have much milder $a$-dependence. As the consequence thermodynamic quantities will also have smaller cutoff dependence if $f_{K}$ is used to set the scale. We checked that the $a$-dependence almost entirely

TABLE V. The values of the coefficients $b_{H}$ and $c_{H}$ entering Eq. (B3) for different hadrons.

\begin{tabular}{lrllll}
\hline \hline & $\rho, \omega$ & \multicolumn{1}{c}{$K^{*}$} & \multicolumn{1}{c}{$\phi$} & \multicolumn{1}{c}{$N$} & \multicolumn{1}{c}{$\Omega$} \\
\hline$b_{H}$ & 1.2138 & 0.259522 & 0.24377 & 1.85148 & 0.306749 \\
$c_{H}$ & 18.1236 & 0 & 0 & 5.42284 & 0 \\
\hline \hline
\end{tabular}

disappears for $K^{*}$ and $\phi$ mesons, as well as for the $\Omega$ baryon if $f_{K}$ is used to set the lattice spacing. However, for the nucleon and other baryons this is not the case. Furthermore, the large taste-symmetry breaking in the ps meson sector cannot be compensated by changing scale from $r_{1}$ to $f_{K}$.

To take into account the effects taste-symmetry breaking in the ps meson sector the contributions of pions, kaons and eta mesons are calculated as [40]

$$
p^{\pi, K, \eta} / T^{4}=\frac{1}{16} \frac{1}{V T^{3}} \sum_{i=0}^{7} d_{\mathrm{ps}_{i}} \ln \mathcal{Z}^{M}\left(m_{\mathrm{ps}_{i}}, T, V\right),
$$

where $m_{\mathrm{ps}_{i}}^{2}=m_{\pi, K, \eta}^{2}+\delta m_{\mathrm{ps}_{i}}^{2}$. The quadratic pseudoscalar meson splittings have been calculated in Ref. [41]. The dependence of these splittings can be fitted well by the form

$$
r_{1} \delta m_{\mathrm{ps}_{i}}^{2}=\frac{e_{i}\left(a / r_{1}\right)^{2}}{1+g_{i}\left(a / r_{1}\right)^{2}} .
$$

The value of the coefficients $e_{i}$ an $g_{i}$ together with the degeneracy factors $d_{\mathrm{ps}_{i}}$ are given in Table VI.

The contribution of the ground state vector mesons can be evaluated at nonzero lattice spacing using Eqs. (B2) and (B3) and the corresponding values of $b_{H}$ and $c_{H}$ from Table V. The $a$-dependence of the octet baryon masses, as well as of $\Omega$ mass is fixed through Eq. (B3) and the values of the coefficients are given in Table V. To completely specify the contribution of the ground state baryons to the partition function we assume that the masses of the decuplet baryons for $S=0,-1$ and -2 have the same $a$-dependence as their octet partners. Thus the contribution of all ground state hadrons at nonzero $a$ is now fixed.

We need to consider also the contributions from the excited mesons and baryons. Unfortunately not much is known about the cutoff dependence of the excited hadron states in the staggered fermion formulations. We will work with two extreme assumptions about the cutoff dependence of the excited hadron states. First, we will assume that the 
TABLE VI. The values of the coefficients $e_{i}$ and $g_{i}$ entering Eq. (B5) for different tastes of ps mesons as well as the degeneracy factors $d_{\mathrm{ps}_{i}}$.

\begin{tabular}{lllcccccc}
\hline \hline $\mathrm{i}$ & 0 & 1 & 2 & 3 & 4 & 5 & 6 & 7 \\
\hline$d_{\mathrm{ps}_{i}}$ & 1 & 1 & 3 & 3 & 3 & 3 & 1 & 1 \\
$e_{i}$ & 0 & 8.34627 & 8.17699 & 14.6245 & 16.0450 & 21.1623 & 23.0067 & 30.8425 \\
$g_{i}$ & 0 & -4.83538 & -6.09594 & -6.72714 & -5.2249 & -6.47337 & -5.49115 & -3.64465 \\
\hline \hline
\end{tabular}

masses of excited hadron states are not affected by the lattice cutoff. Second, we will assume that the masses of the excited hadron states are affected by the lattice cutoff the same way as the masses of the corresponding ground state hadrons. Furthermore, we will calculate the EoS in the HRG model assuming that only ps mesons are affected by the taste-symmetry breaking. We will compare these three scenarios with the continuum HRG model in order to understand the size of the cutoff effects. We will use the HRG model with missing states (HRG-QM) in what follows. The trace anomaly calculated for different $N_{\tau}$ is shown in Fig. 15 and compared to the lattice results. In Fig. 16 we show the pressure calculated for the same set of $N_{\tau}$ values. We see that the difference between the continuum HRG and the lattice HRG is larger for the pressure than for the trace anomaly, and the continuum limit is approached from below. The difference in the cutoff dependence of the trace anomaly and the pressure can be understood as follows. The cutoff effects make the hadrons heavier. This reduces the pressure as expected. However, states with larger masses contribute more to the trace anomaly. So this partially compensates the exponential suppression due to larger quark masses in the case of the trace anomaly in the considered temperature range. At sufficiently low temperatures, the cutoff dependence of the pressure and the trace anomaly are qualitatively similar. We also note that the reduction of the pressure relative to the continuum HRG expectation is mostly due to the ps meson sector. As one can see from Fig. 16 taking into account the modification of the baryon and vector meson masses in the
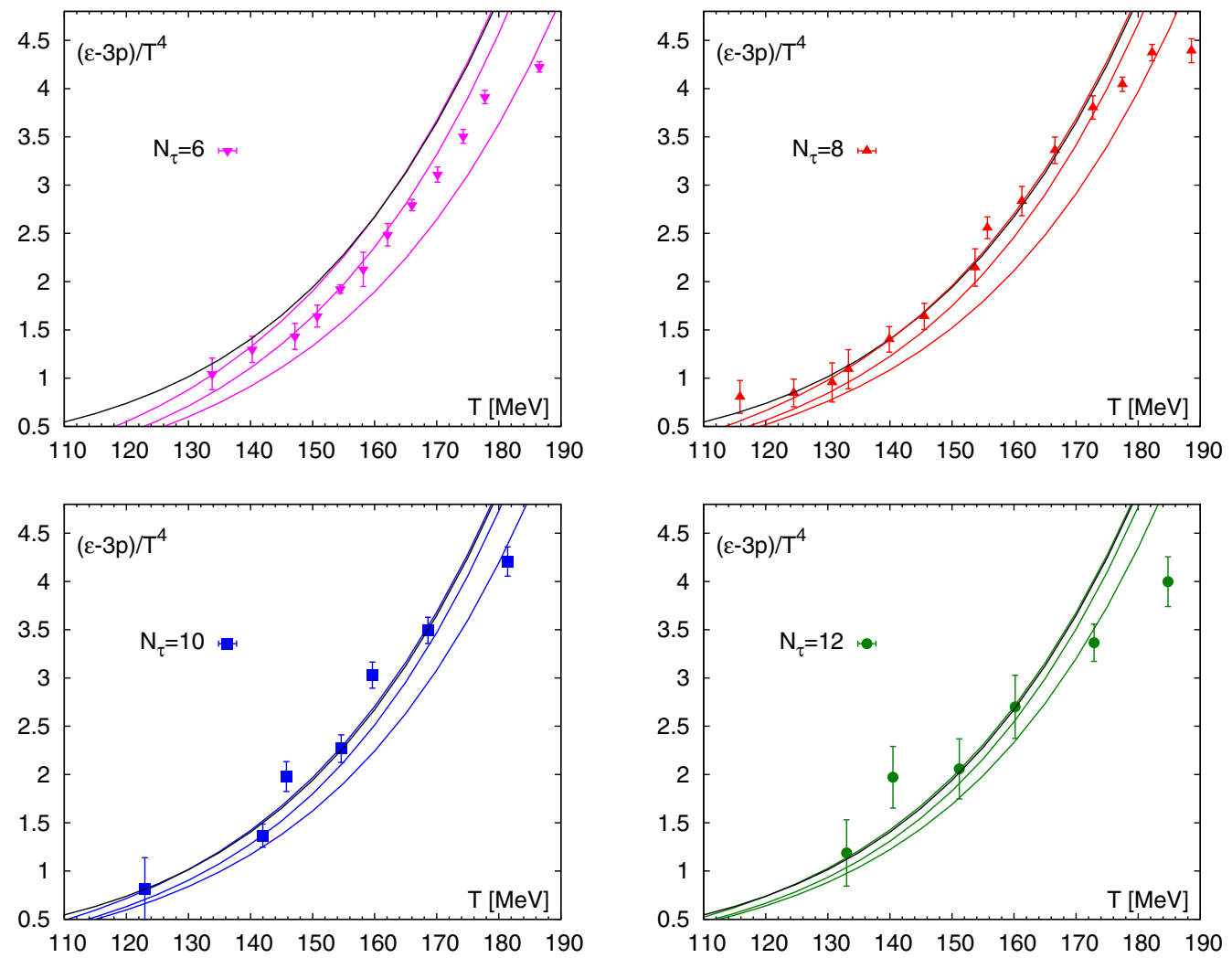

FIG. 15. The trace anomaly calculated in the HRG-QM and in the HRG-QM with modified hadron spectrum for $N_{\tau}=6,8,10$ and 12 , and compared with the lattice results, see Sec. III for details. The solid thick line corresponds to the continuum HRG-QM, while the top, middle and bottom colored thin lines correspond to the lattice HRG-QM, where only the ps mesons are modified, all ground states hadron are modified, and all ground state and excited state hadrons are modified, respectively. 

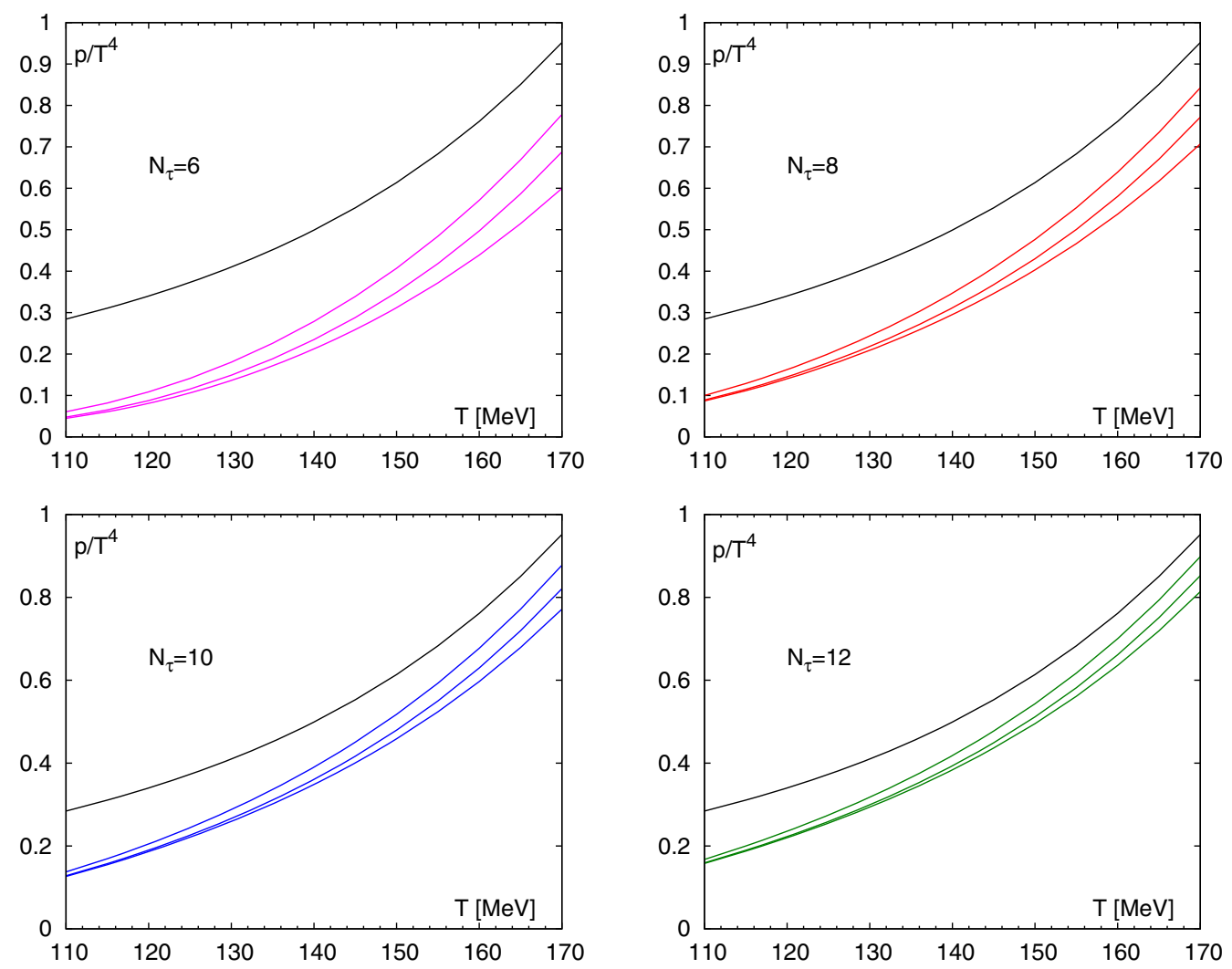

FIG. 16. The pressure calculated in the HRG-QM and in the HRG-QM with modified hadron spectrum for $N_{\tau}=6,8,10$ and 12 from top left to bottom right. The solid thick line corresponds to the continuum HRG-QM. The top, middle and bottom colored thin lines correspond to the lattice HRG-QM, where only the ps mesons are modified, all ground states hadron are modified and all the ground state and excited state hadrons are modified, respectively.

HRG calculations only results in relatively small effects. We also note that for $\mathrm{p} 4$ and asqtad actions the cutoff effects due to taste-symmetry breaking are much larger [40].

We use the value of the pressure in the modified HRG$\mathrm{QM}$, in which the cutoff dependence of all the ground state hadrons is taken into account as discussed above (middle curves in Fig. 16) to determine the pressure at some initial value of the temperature $T_{0}$ in the integral method (see Sec. IV). To estimate the uncertainty in $p\left(T_{0}\right)$ we consider the difference between the HRG-QM model in which only ps mesons are modified (upper curves in Fig. 16) and the HRG-QM model in which all ground state and excited state hadrons are modified (lower curves in Fig. 16). The resulting values are given in Table II.
[1] Y. Aoki, Z. Fodor, S. D. Katz, and K. K. Szabo, J. High Energy Phys. 01 (2006) 089.

[2] C. Bernard, T. Burch, C. E. DeTar, S. Gottlieb, L. Levkova, U. M. Heller, J. E. Hetrick, R. Sugar, and D. Toussaint, Phys. Rev. D 75, 094505 (2007).

[3] M. Cheng et al., Phys. Rev. D 77, 014511 (2008).

[4] A. Bazavov et al., Phys. Rev. D 80, 014504 (2009).

[5] M. Cheng et al., Phys. Rev. D 81, 054504 (2010).

[6] S. Borsanyi, G. Endrodi, Z. Fodor, A. Jakovac, S. D. Katz, S. Krieg, C. Ratti, and K. K. Szabo, J. High Energy Phys. 11 (2010) 077.
[7] S. Borsanyi, Z. Fodor, C. Hoelbling, S. D. Katz, S. Krieg, and K. K. Szabo, Phys. Lett. B 730, 99 (2014).

[8] A. Bazavov et al. (HotQCD Collaboration), Phys. Rev. D 90, 094503 (2014).

[9] S. Borsanyi et al., Nature (London) 539, 69 (2016).

[10] M. Laine and Y. Schroder, Phys. Rev. D 73, 085009 (2006).

[11] G. Boyd, J. Engels, F. Karsch, E. Laermann, C. Legeland, M. Lutgemeier, and B. Petersson, Nucl. Phys. B469, 419 (1996).

[12] B. Beinlich, F. Karsch, and E. Laermann, Nucl. Phys. B462, 415 (1996). 
[13] U. M. Heller, F. Karsch, and B. Sturm, Phys. Rev. D 60, 114502 (1999).

[14] A. Bazavov et al. (MILC Collaboration), Proc. Sci., LATTICE2010 (2010) 074, [arXiv:1012.0868].

[15] A. Bazavov, N. Brambilla, H. T. Ding, P. Petreczky, H. P. Schadler, A. Vairo, and J. H. Weber, Phys. Rev. D 93, 114502 (2016).

[16] A. Bazavov, N. Brambilla, P. Petreczky, A. Vairo, and J. H. Weber, Technical University of Munich, Report No. TUMEFT 81/16.

[17] B. Beinlich, F. Karsch, E. Laermann, and A. Peikert, Eur. Phys. J. C 6, 133 (1999).

[18] P. Hegde, F. Karsch, E. Laermann, and S. Shcheredin, Eur. Phys. J. C 55, 423 (2008).

[19] A. Bazavov, H. T. Ding, P. Hegde, F. Karsch, C. Miao, S. Mukherjee, P. Petreczky, C. Schmidt, and A. Velytsky, Phys. Rev. D 88, 094021 (2013).

[20] H. T. Ding, S. Mukherjee, H. Ohno, P. Petreczky, and H. P. Schadler, Phys. Rev. D 92, 074043 (2015).

[21] A. Bazavov et al. (HotQCD Collaboration), Phys. Rev. D 86, 034509 (2012).

[22] N. Haque, A. Bandyopadhyay, J. O. Andersen, M. G. Mustafa, M. Strickland, and N. Su, J. High Energy Phys. 05 (2014) 027.

[23] A. Rebhan, in Proceedings, 5th International Conference on Strong and Electroweak Matter (SEWM 2002): Heidelberg, Germany, October 2-5, 2002 (World Scientific, Singapore, 2003), p. 157 [arXiv:hep-ph/0301130].
[24] J. P. Blaizot, E. Iancu, and A. Rebhan, Phys. Rev. Lett. 83, 2906 (1999).

[25] J. P. Blaizot, E. Iancu, and A. Rebhan, Phys. Lett. B 470, 181 (1999).

[26] J. P. Blaizot, E. Iancu, and A. Rebhan, Phys. Rev. D 63, 065003 (2001).

[27] J. P. Blaizot, E. Iancu, and A. Rebhan, Phys. Lett. B 523, 143 (2001).

[28] MILC code, http://www.physics.utah.edu/ detar/milc/.

[29] R statistical package, http://www.r-project.org/.

[30] C. R. Allton, Nucl. Phys. B, Proc. Suppl. 53, 867 (1997).

[31] S. Capstick and N. Isgur, Phys. Rev. D 34, 2809 (1986).

[32] R. G. Edwards, N. Mathur, D. G. Richards, and S. J. Wallace (Hadron Spectrum), Phys. Rev. D 87, 054506 (2013).

[33] A. Majumder and B. Muller, Phys. Rev. Lett. 105, 252002 (2010).

[34] A. Bazavov et al., Phys. Rev. Lett. 113, 072001 (2014).

[35] A. Bazavov et al., Phys. Lett. B 737, 210 (2014).

[36] A. Bazavov et al., Phys. Rev. D 95, 054504 (2017).

[37] A. Bazavov et al. (HotQCD Collaboration), Phys. Rev. D 96, 074510 (2017).

[38] U. Loring, B. C. Metsch, and H. R. Petry, Eur. Phys. J. A 10, 395 (2001).

[39] U. Loring, B. C. Metsch, and H. R. Petry, Eur. Phys. J. A 10, 447 (2001).

[40] P. Huovinen and P. Petreczky, Nucl. Phys. A837, 26 (2010).

[41] A. Bazavov, T. Bhattacharya, M. Cheng, C. DeTar, H. Ding et al., Phys. Rev. D 85, 054503 (2012). 